\title{
Hyponatremia and Hyporeninemic-Hypoaldosteronism in a Pediatric Intensive Care Unit Patient
}

\author{
Rafael Lemus ${ }^{\mathrm{a}, \mathrm{c}}$, Joseph D. Tobias ${ }^{\mathrm{b}}$
}

\begin{abstract}
Hyponatremia is one of the most commonly encountered electrolyte abnormalities encountered in the pediatric world. Defined as a serum or plasma sodium less than $135 \mathrm{mEq} / \mathrm{L}$, the etiology of hyponatremia is one that can typically be determined by performance of a thorough history. However, occasionally the etiology of a patient's hyponatremia is more elusive and determined only after laboratory evaluation. We present a 6-year-old girl with a complex medical history including spinal muscular atrophy, tracheostomy and ventilator dependence, who was admitted to the pediatric intensive care unit for treatment and evaluation of seizures with hyponatremia that was initially thought to be due to syndrome of inappropriate anti-diuretic hormone or cerebral salt wasting. However, during her hospital course, it was determined that the hyponatremia was more indicative of a rarer and much less common cause of hyponatremia, hyporeninemic-hypoaldosteronism. The physiological factors controlling serum sodium are reviewed, the etiologies of hyponatremia are presented and the treatment of hyporeninemic-hypoaldosteronism is discussed.
\end{abstract}

Keywords: Hyponatremia; Renin; Aldosterone; Sodium

\section{Introduction}

Hyponatremia, defined as a serum sodium level less than 135 $\mathrm{mEq} / \mathrm{L}$, is one of the most common electrolyte disturbances noted in the pediatric-aged patient [1]. It may be found incidentally on a routine electrolyte panel in otherwise asymptomatic patients. However, when there is an abrupt change in the serum sodium concentration or as levels fall below $125 \mathrm{mEq} / \mathrm{L}$, non-specific symptoms such as nausea, headache, malaise and

\footnotetext{
Manuscript submitted March 23, 2020, accepted April 8, 2020

aDepartment of Pediatrics, Nationwide Children's Hospital and The Ohio State University College of Medicine, Columbus, OH, USA

bDepartment of Anesthesiology \& Pain Medicine, Nationwide Children's Hospital and The Ohio State University College of Medicine, Columbus, OH, USA

${ }^{\mathrm{c} C o r r e s p o n d i n g ~ A u t h o r: ~ R a f a e l ~ L e m u s, ~ D e p a r t m e n t ~ o f ~ P e d i a t r i c s, ~ N a t i o n-~}$ wide Children's Hospital, 700 Children's Drive, Columbus, OH 43205, USA. Email: Rafael.Lemus@nationwidechildrens.org
}

doi: https://doi.org/10.14740/jmc3457 even neurologic symptoms including seizures may occur [2]. When hyponatremia is detected, determining the etiology and identifying the appropriate treatment plan can prevent further decreases in serum and end-organ consequences including seizures. We present a 6-year-old girl with a complex medical history including spinal muscular atropy (SMA), tracheostomy and ventilator dependence, who was admitted to the pediatric intensive care unit (PICU) for the treatment and evaluation of seizures with hyponatremia that was initially thought to be due to either the syndrome of inappropriate anti-diuretic hormone (SIADH) or cerebral salt wasting. However, during her course in the PICU, it was determined that the hyponatremia was more indicative of a rarer and much less common cause of hyponatremia, hyporeninemic-hypoaldosteronism (HH). The physiological factors controlling serum sodium are reviewed, potential etiologies of hyponatremia are presented and the pathophysiology of $\mathrm{HH}$ is discussed.

\section{Case Report}

Review of this case and presentation in this format was in accordance with the guidelines of the Institutional Review Board at Nationwide Children's Hospital (Columbus, OH). The patient was a 6-year-old, 22.7-kg female with SMA and tracheostomy with ventilator dependence who was admitted with concerns for seizures. She was in her usual state of health and had no recent changes in activity, fevers, nausea, vomiting, diarrhea or difficulty tolerating home feeds. Of note, she did have a history of one prior seizure 3 years ago that was related to hyponatremia, of which she had been maintained on sodium supplements at home upon discharge. On arrival to the emergency department (ED), she had active tonic-clonic seizure activity and was treated with intranasal midazolam (two doses) followed by a loading dose of fos-phenytoin which resulted in control of the seizure activity. Laboratory evaluation revealed normal electrolytes (serum sodium $140 \mathrm{mEq} / \mathrm{L}$ ), glucose, blood urea nitrogen, creatinine, lactic acid, liver function tests and complete blood count. Imaging included a normal chest radiograph and computed tomography of the head which was negative except for mild volume loss in the frontal lobes and middle ear fluid bilaterally. Arterial blood gas analysis was remarkable for respiratory acidosis thought to be secondary to carbon dioxide retention in the setting of seizures which improved with ventilator adjustments. Due to the seizures of uncertain etiology, broad spectrum antibiotics (amikacin, cefepime and vancomycin) were started and she was admitted to 
the PICU. Approximately $20 \mathrm{~h}$ after admission, the patient had another seizure and repeat laboratory evaluation was remarkable for hyponatremia (serum sodium $113 \mathrm{mEq} / \mathrm{L}$ ). During this time, she was fluid positive by $900 \mathrm{~mL}$ and the hyponatremia was initially thought to be due to SIADH. Hyponatremia was treated with 3\% normal saline and fluid restriction which increased the serum sodium from 113 to $130 \mathrm{mEq} / \mathrm{L}$ over the ensuing $12 \mathrm{~h}$. A respiratory viral panel was positive for respiratory syncytial virus (RSV). While her initial seizure was thought to be secondary to hypoxemia due to respiratory distress from an RSV infection and not related to hyponatremia as the initial serum sodium was $140 \mathrm{mEq} / \mathrm{L}$, with her subsequent seizures occurring in the setting of profound hyponatremia, the preliminary diagnosis was SIADH due to an acute critical illness. However, this diagnosis was not definitive as the patient had had a brisk urine output and persistent hyponatremia (serum sodium $113-118 \mathrm{mEq} / \mathrm{L}$ ) despite fluid restriction and the administration of $3 \%$ hypertonic saline. Therefore, an ongoing workup for the hyponatremia was pursued to identify other causes of hyponatremia including cerebral salt wasting and excessive renal losses of sodium including measurement of serum cortisol, aldosterone, renin and thyroid hormones. A serum cortisol and thyroid function tests were normal. Serum osmolality was decreased $(249 \mathrm{mOsm} / \mathrm{kg}$, normal 270 - 310) with an elevated urine sodium $(168 \mathrm{mEq} / \mathrm{L})$ and a normal urine osmolality (445 $\mathrm{mOsm} / \mathrm{kg})$. With the ongoing urinary losses of sodium, hyponatremia recurred and enteral sodium supplementation was started $(50 \mathrm{mEq}$ every $6 \mathrm{~h}$ via Gtube). Further workup revealed a renin level of $0.74 \mathrm{ng} / \mathrm{dL} / \mathrm{h}$ (normal: 0.50 - 5.85 for 5 - 9 years old) and an aldosterone level of less than $1 \mathrm{ng} / \mathrm{dL}$. Given the severely depressed aldosterone level and the low normal renin level in the setting of hyponatremia, $\mathrm{HH}$ was postulated as the etiology of the hyponatremia. Throughout the course of her hospital stay, the hyponatremia was controlled with enteral sodium supplementation. Once the increased ventilator settings due to RSV infection resolved, she was discharged home. She returned back to her baseline behavior and neurologic status after resolution of the hyponatremia and seizure activity. On follow-up with the pediatric nephrology service 3 months after her hospital admission, her serum sodium remained normal with enteral sodium supplementation. However, over the ensuing months, she would have episodes of hyponatremia during acute illnesses. Hyponatremia was treated with increased enteral and intravenous supplementation and the sodium wasting resolved as the acute illness improved.

\section{Discussion}

The serum sodium concentration is maintained within the plasma at a range of $135-145 \mathrm{mEq} / \mathrm{L}$ through the thirst mechanism, free water and sodium intake, balanced by free water and sodium excretion $[3,4]$. The process is regulated by key hormonal factors which control serum sodium, primarily antidiuretic hormone $(\mathrm{ADH})$ but also the renin and aldosterone systems, coupled with the renal handling of sodium and free water. An imbalance or dysfunction of any of these processes can lead to derangements of serum sodium. In the absence of confounding factors such as hyperglycemia or unmeasured osmoles (mannitol, alcohols), serum osmolality is primarily de- termined by the serum sodium concentration. In the presence of hypovolemia or hypernatremia, the thirst mechanism driven by various factors such as ADH and the renin-angiotensin system and by osmolality receptors located in the hypothalamus control the thirst mechanism to increase or decrease the intake of free water to normalize serum sodium. The ADH system also plays a key role in regulating serum sodium and free water excretion through the kidneys. In the setting of a low serum sodium and low serum osmolality, ADH release is suppressed, leading to increased renal tubular losses of free water.

Hyponatremia may result from several physiologic derangements related to either a lack of serum sodium, excessive water in relationship to sodium or a combination of the two. It may occur in the setting of hypovolemia, normovolemia, or hypervolemia relative to the concentration of sodium in relationship to free water. Dehydration with excessive intake of free water or the inappropriate administration of hypotonic fluids following surgery and SIADH related to acute illnesses predominate as the primary causes of hyponatremia in the hospitalized patient [5]. However, when treatment processes aimed at these conditions (fluid restriction or the administration of additional sodium) fail to correct serum sodium values, a more thorough evaluation may be indicated. Such was the case in our patient who was initially thought to have SIADH.

Hyponatremia may also occur due to excessive renal losses of sodium related to renal tubular issues or endocrine disturbances. When evaluating the hospitalized patient with hyponatremia, measuring the urinary sodium concentration may be helpful in determining the primary pathologic processes responsible for hyponatremia. In the absence of clinical signs suggestive of SIADH (relative oliguria), a high urinary sodium concentration in the setting of hyponatremia is suggestive of excessive urinary losses related to either renal tubular, central nervous system or endocrine issues such as cerebral salt wasting, glucocorticoid deficiency, hypothyroidism, or renal salt related to renal tubular dysfunction. In our patient, given her primary disease processes, cerebral salt wasting seemed unlikely. Thyroid function and a random serum cortisol value were normal thereby leading us to consider other rarer conditions including a disorder of the renin-angiotensin system, $\mathrm{HH}$.

Renin production from the juxtaglomerular apparatus in the kidneys can be stimulated by either hypotension or electrolyte changes (low sodium, potassium, or chloride) concentrations in the distal tubules [6]. Renin cleaves angiotensinogen, which is produced in the liver, to angiotensin I, which is then converted in the lungs by angiotensin converting enzymes (ACE) to angiotensin II. Angiotensin II is a potent vasoconstrictor and also stimulates the release of aldosterone from the adrenal gland cortex [3, 4]. Angiotensin II increases serum sodium by acting on the renal tubules to increase sodium-potassium exchange and on the zona glomerulosa of the adrenal cortex to stimulate the release of aldosterone, which will in turn increase renal sodium resorption and potassium excretion in the distal tubules of the nephron.

$\mathrm{HH}$ results in diminished renin and aldosterone release even in the setting of hyponatremia or hyperkalemia [7-9]. The lower aldosterone levels result in persistent urinary losses of sodium and the high urine sodium concentration as noted in our patient despite hyponatremia. $\mathrm{HH}$ has been reported most com- 
monly in the adult population in setting of diabetic nephropathy, chronic interstitial nephritis or in patients taking medications such as nonsteroidal anti-inflammatory drugs or calcineurin inhibitors. Rarely congenital enzymatic disorders affecting the aldosterone system may result in a low aldosterone state, generally resulting in hyperkalemia as a clinical manifestation [10].

In the evaluation of our patient, despite persistent hyponatremia, we noted elevated urinary concentrations of sodium coupled with a low aldosterone level and low-normal renin level. Although we postulate $\mathrm{HH}$ as the etiology of these issues, hyperkalemia which was not seen in our patient is also commonly present. The pathologic process appeared to be transient occurring only during periods of acute illness as our patient's serum sodium tended to normalize when she was healthy and hyponatremia recurred when she was acutely ill. Potential mechanisms for the $\mathrm{HH}$ noted in our patient include disruption of aldosterone synthesis from acute illness suppression of synthetic function of the zona glomerulosa of the adrenal cortex, pro-inflammatory cytokine inhibition of aldosterone release, or transient dysfunction of the juxtaglomerular cells of the kidneys related to alterations in perfusion or tissue oxygen delivery. Typically, the mainstay therapy for patients with $\mathrm{HH}$ is removal of offending pharmacologic agents and supplementation with fludrocortisone. Our patient was treated solely with sodium supplementation which resulted in correction and subsequent control of her serum sodium concentrations.

In summary, while there are numerous potential etiologies for hyponatremia in the critically ill patient, an elevated urinary sodium in the presence of hyponatremia suggests deficient renal handling of sodium related either to a primary renal tubular problem or endocrine dysfunction. While relative oliguria may point to the diagnosis of SIADH, a normal or elevated urine output should lead one to a more thorough elevation of potential etiologies including cerebral salt wasting, primary renal tubular dysfunction or endocrine disturbances. The latter may include thyroid dysfunction, adrenal insufficiency or primary disorders of the renin-angiotensin system.

\section{Acknowledgments}

None to declare.

\section{Financial Disclosure}

None to declare.

\section{Conflict of Interest}

None to declare.

\section{Informed Consent}

In accordance with IRB of Nationwide Children's Hospital, need for documentation of informed consent waved.

\section{Author Contributions}

Rafael Lemus: review of case, preparation of initial and subsequent drafts. Joseph Tobias: concept, care of patient, review of all drafts.

\section{Data Availability}

The authors declare that data supporting the findings of this study are available within the article.

\section{References}

1. Reid-Adam J. Hyponatremia. Pediatr Rev. 2013;34(9):417419.

2. Rodriguez MJ, Alcaraz A, Solana MJ, Garcia A. Neurological symptoms in hospitalised patients: do we assess hyponatraemia with sufficient care? Acta Paediatr. 2014;103(1):e7-e10.

3. Berry PL, Belsha CW. Hyponatremia. Pediatr Clin North Am. 1990;37(2):351-363.

4. Sterns RH, Hix JK, Silver SM. Management of hyponatremia in the ICU. Chest. 2013;144(2):672-679.

5. Wang J, Xu E, Xiao Y. Isotonic versus hypotonic maintenance IV fluids in hospitalized children: a meta-analysis. Pediatrics. 2014;133(1):105-113.

6. Kobori H, Nangaku M, Navar LG, Nishiyama A. The intrarenal renin-angiotensin system: from physiology to the pathobiology of hypertension and kidney disease. Pharmacol Rev. 2007;59(3):251-287.

7. Phelps KR, Lieberman RL, Oh MS, Carroll HJ. Pathophysiology of the syndrome of hyporeninemic hypoaldosteronism. Metabolism. 1980;29(2):186-199.

8. Sousa AG, Cabral JV, El-Feghaly WB, de Sousa LS, Nunes AB. Hyporeninemic hypoaldosteronism and diabetes mellitus: Pathophysiology assumptions, clinical aspects and implications for management. World J Diabetes. 2016;7(5):101-111.

9. DeFronzo RA. Hyperkalemia and hyporeninemic hypoaldosteronism. Kidney Int. 1980;17(1):118-134.

10. Peter M, Sippell WG. Congenital hypoaldosteronism: the Visser-Cost syndrome revisited. Pediatr Res. 1996;39(3):554-560. 